\title{
MicroRNA-330-5p inhibits osteosarcoma cell growth and invasion by targeting the proto-oncogene survivin
}

\author{
HAO WANG ${ }^{1 *}$, LEI LIU ${ }^{2 *}$ and SHIYUAN FANG ${ }^{1}$ \\ ${ }^{1}$ Department of Traumatic Orthopaedics, Anhui Provincial Hospital, Anhui Medical University; \\ ${ }^{2}$ Department of Traumatic Orthopaedics, The First Affiliated Hospital of USTC, \\ Anhui Provincial Hospital, Hefei, Anhui 230000, P.R. China
}

Received May 20, 2018; Accepted December 10, 2018

DOI: $10.3892 / \mathrm{mmr} .2019 .10447$

\begin{abstract}
Increasing evidence has suggested the crucial role of the dysregulation of microRNAs (miRNAs) in osteosarcoma (OS) progression. MicroRNA (miR)-330-5p has been reported to exert tumor suppressive effects in various types of human cancer. However, the role of miR-330-5p in the development of OS and the underlying mechanism remain to be clarified. In the present study, miR-330-5p expression was found to be significantly decreased in OS tissues and cell lines. In addition, low miR-330-5p expression was highly correlated with the overall survival and clinical stage of OS. Overexpression of miR-330-5p inhibited the viability, migration and invasion, and promoted the apoptosis of OS cells, as well as induced cell cycle arrest at the $\mathrm{G}_{2} / \mathrm{M}$ phase. Subsequently, the proto-oncogene survivin was identified as a functional target of miR-330-5p, and this was validated using a luciferase reporter assay. It was also demonstrated that survivin expression was markedly increased in OS tissues, and that it was negatively correlated with the expression of miR-330-5p. Furthermore, overexpression of survivin significantly abrogated the tumor-suppressive effect induced by miR-330-5p on OS cells. In conclusion, these results revealed that the miR-330-5p/survivin axis has a significant tumor-suppressive effect on OS, and may serve as a diagnostic and therapeutic target for the treatment of OS.
\end{abstract}

\section{Introduction}

Osteosarcoma (OS) is a primary bone malignancy that mainly occurs in young adults and adolescents (1). In spite of recent developments in combinational chemotherapy and surgical techniques, the long-term survival of OS patients

Correspondence to: Dr Shiyuan Fang, Department of Traumatic Orthopaedics, Anhui Provincial Hospital, Anhui Medical University, 17 Lujiang Road, Hefei, Anhui 230000, P.R. China

E-mail: shhiyuanfang@163.com

${ }^{*}$ Contributed equally

Key words: osteosarcoma, microRNA-330-5p, survivin remains unsatisfactory, with metastatic and recurrent disease observed (2). Thus, there is an urgent need for exploring the pathogenesis of OS, and identifying novel and efficient biomarkers for predicting the prognosis of OS patients.

MicroRNAs (miRNAs) are a type of highly conserved noncoding RNAs with a length of 18-25 nucleotides, which regulate gene expression by binding to the 3 '-untranslated regions (UTRs) of their target mRNA in order to repress transcription or induce mRNA degradation $(3,4)$. Currently, growing evidence suggests that the involvement of miRNAs in multiple cellular processes, including cell apoptosis, proliferation and metastasis $(5,6)$. Dysregulated miRNAs may contribute to the development of numerous types of human cancer, including OS. For instance, an evident increase in miR-378 was reported in OS tissues and cells, while miR-378 overexpression promoted the proliferation of OS cells by targeting Krüppel-like factor 9 (7). Liu et al (8) reported decreased expression of miR-935 in OS tissues, and restoration of this expression evidently restricted cell proliferation and invasion. In addition, the study by Yang et al (9) demonstrated that miR-183 suppressed the LDL receptor-related protein $6 / \mathrm{Wnt} / \beta$-catenin signaling and, thereby, inhibited MG63 cell growth, migration and invasion in vivo and in vitro. Furthermore, Dong et al (10) reported that miR-223 was markedly decreased in the serum of OS patients, suggesting that miR-223 may serve as a potential diagnostic and prognostic biomarker of OS.

The aberrant expression of miR-330-5p has been reported in certain types of cancer, including glioblastoma (11) and pancreatic cancer (12). A study by Wang et al (13) revealed that high miR-330-5p expression was correlated with worse prognosis in patients with breast cancer. It was also reported that miR-330-5p was significantly decreased in cutaneous malignant melanoma (CMM) tissues, and forced expression of miR-330-5p suppressed CMM cell proliferation and invasion (14). In addition, Wei et al (15) demonstrated that miR-330-5p functioned as an oncogene in non-small cell lung cancer (NSCLC) through activating the mitogen-activated protein kinase/extracellular signal-regulated kinase (ERK) signaling pathway. Nevertheless, the biological function and clinical value of miR-330-5p in OS remain to be investigated.

In the present study, the expression levels of miR-330-5p in OS tissues and cell lines were investigated, and the correlation between miR-330-5p expression and the clinicopathological 
characteristics of patients was then analyzed. The study also investigated the effects of miR-330-5p expression on the proliferation, invasion, apoptosis and cell cycle distribution of OS cells. In addition, the regulatory mechanisms of miR-330-5p on OS cells, as well as the potential relationship between miR-330-5p and proto-oncogene survivin (also known as baculoviral IAP repeat-containing protein 5) were investigated. The study findings provide novel insights into the role of miR-330-5p in the development of OS.

\section{Materials and methods}

Patients and samples. A total of 63 surgically resected OS tissue specimens were acquired from patients with OS at the Department of Traumatic Orthopaedics at Anhui Provincial Hospital, Anhui Medical University (Hefei, China) between January 2012 and December 2016. The patients were assigned into two groups according to the presence or absence of metastasis, as determined by radiology. The clinicopathological data of the patients are shown in Table I. The clinical stage of the patients was classified according to the Tumor Node Metastasis (TNM) Classification of Malignant Tumors (Sixth edition) from the Union for International Cancer Control (10). In addition, 20 osteochondroma (a benign bone lesion) tumor tissue samples from amputees were selected and served as the Control group; the Control group included 9 males and 11 females, whose ages ranged between 10 and 57 with a mean age of $25.93 \pm 10.57$. The present study was approved by the Institutional Ethics Review Board of Anhui Provincial Hospital, Anhui Medical University, and informed consent was obtained from adult participant or from the legal guardians of participants $<18$ years old prior to participation in this study. The samples were immediately snap-frozen in liquid nitrogen and stored at $-80^{\circ} \mathrm{C}$ until further use.

Cell culture. The OS-derived human cell lines HOS, U2OS and MG63, and the conditionally immortalized human fetal osteoblastic cell line hFOB1.19 were purchased from the Shanghai Cell Bank of the Chinese Academy of Sciences (Shanghai, China). The 293T cell line was obtained from the American Type Culture Collection (Manassas, VA, USA). HOS, U2OS, MG63 and 293T cells were maintained in Dulbecco's modified Eagle's medium (DMEM; Gibco; Thermo Fisher Scientific, Inc., Waltham, MA, USA) supplemented with $10 \%$ fetal bovine serum (FBS; Gibco; Thermo Fisher Scientific, Inc.). The hFOB1.19 cells were maintained in a 1:1 mixture of Ham's F12 medium and DMEM supplemented with $2.5 \mathrm{ml}$ glutamine (without phenol red) and $10 \%$ FBS. All cells were incubated in a humidified atmosphere containing $5 \% \mathrm{CO}_{2}$ at $37^{\circ} \mathrm{C}$.

Reverse transcription-quantitative polymerase chain reaction $(R T-q P C R)$. Total RNA was extracted from tissues or cells using TRIzol ${ }^{\circledR}$ reagent (Thermo Fisher Scientific, Inc.) and miRNA was extracted using the mirVana RNA isolation kit (Ambion; Thermo Fisher Scientific, Inc.), according to the manufacturer's protocols. RNA concentration was measured using NanoDrop ND-1000 (Thermo Fisher Scientific, Inc., Wilmington, DE, USA). RT reactions for miRNA detection were performed using a miRNA Reverse Transcription kit (Qiagen, Inc., Valencia, CA, USA), whereas RT reactions for mRNA detection were conducted using the Takara PrimeScript ${ }^{\mathrm{TM}}$ First Strand cDNA Synthesis (Takara Biotechnology Co., Ltd., Dalian, China). Subsequently, an ABI 7500 system (Thermo Fisher Scientific, Inc.) was used to conduct qPCR using a standard protocol described in the SYBR Green PCR kit (Toyobo Life Science, Osaka, Japan). Briefly, the amplification protocol was set as follows: Initial denaturation at $95^{\circ} \mathrm{C}$ for $5 \mathrm{~min}$; followed by 30 cycles of denaturation at $94^{\circ} \mathrm{C}$ for $15 \mathrm{sec}$, annealing at $55^{\circ} \mathrm{C}$ for $30 \mathrm{sec}$ and extension at $70^{\circ} \mathrm{C}$ for $30 \mathrm{sec}$. U6 and GAPDH were used as the internal references and to normalize expression data for miR-330-5p and survivin levels, respectively. The sequences of the primers were as follows: miR-330-5p, forward 5'-TCTCTGGGCCTGTGTCTT AGGC-3', reverse 5'-GCTATCTCAGGGCTTGTTGCTTCA GTCCTCCTGGG-3'; U6, forward 5'-TGCGGGTGCTCGCTT CGCAGC-3', reverse 5'-CCAGTGCAGGGTCCGAGGT-3'; survivin, forward 5'-GCACTTTCTTCGCAGTTTC-3', reverse 5'-GTGAGGTGTGCTGTTCGAGA-3'; GAPDH, forward 5'-AGGTCGGTGTGAACGGATTTG-3', reverse 5'-TGTAGA CCATGTAGTTGAGGTCA-3'. The relative expression levels were calculated using the $2^{-\Delta \Delta \mathrm{Cq}}$ method (16).

Cell transfection. miR-330-5p mimics (5'-TCTCTGGGCCTG TGTCTTAGGC-3'), negative control mimics (mimics NC; 5'-GCCTAAGACACAGGCCCAGAGA-3'), miR-330-5p inhibitor and inhibitor NC were purchased from GenePharma Co., Ltd. (Shanghai, China). In addition, the coding domain sequences of survivin mRNA were amplified by PCR and inserted into a pcDNA3.0 overexpression vector (pcDNA-survivin; Invitrogen; Thermo Fisher Scientific, Inc.). Cells were cultured to $80 \%$ confluence and subsequently transfected with miR-330-5p mimics (50 $\mathrm{nM})$, control mimics (50 nM), miR-330-5p inhibitor $(50 \mathrm{nM})$ or control inhibitor $(50 \mathrm{nM})$ using Lipofectamine ${ }^{\circledR} 2000$ reagent (Invitrogen; Thermo Fisher Scientific, Inc.), according to a previous study (11). pcDNA-survivin $(2 \mu \mathrm{g})$ and the control empty plasmid pcDNA3.0 $(2 \mu \mathrm{g})$ was transfected into OS cells with Lipofectamine 2000, following manufacturer's protocol. Transfected cells were incubated in a $37^{\circ} \mathrm{C}$ incubator with $5 \%$ $\mathrm{CO}_{2}$ for 24,48 or $72 \mathrm{~h}$. For cell viability assay, cell apoptosis assay and cell cycle distribution assay, cells were harvested for further experiments after $48 \mathrm{~h}$ of transfection at $37^{\circ} \mathrm{C}$. Cell invasion assay and wound-healing assay were performed after $24 \mathrm{~h}$ of transfection at $37^{\circ} \mathrm{C}$, and images of the stained cells were captured at the next $24 \mathrm{~h}$.

Cell viability assay. For the cell viability assay, U2OS and MG63 cells were seeded in a 96-well plate at the density of $5 \times 10^{3}$ cells per well and transfected with the miR-330-5p mimics, miR-330-5p inhibitor or pcDNA-survivin. After 24,48 or $72 \mathrm{~h}$ of incubation, the relative viability of the cells was determined using a Cell Counting Kit-8 (CCK-8) assay (Beyotime Institute of Biotechnology, Jiangsu, China). Briefly, $10 \mu \mathrm{l} \mathrm{CCK-8} \mathrm{solution}$ was added to each well and incubated at $37^{\circ} \mathrm{C}$ in a $5 \% \mathrm{CO}_{2}$ cell incubator for $90 \mathrm{~min}$. Next, the absorbance rates were measured at $450 \mathrm{~nm}$ with a SpectraMax M5 reader (Molecular Devices, LLC, Shanghai, China). All experiments were performed in triplicate.

Cell apoptosis assay. After $48 \mathrm{~h}$ of transfection, an Annexin V-fluorescein isothiocyanate (FITC) Apoptosis Detection kit (Abcam, Cambridge, UK) was used to detect cell 
Table I. Association between miR-330-5p expression and clinicopathological features of osteosarcoma patients.

miR-330-5p expression

\begin{tabular}{|c|c|c|c|c|}
\hline Clinicopathological feature & No. of cases & High $(n=25)$ & Low $(n=38)$ & P-value \\
\hline Sex & & & & 0.961 \\
\hline Male & 33 & 13 & 20 & \\
\hline Female & 30 & 12 & 18 & \\
\hline Age (years) & & & & 0.493 \\
\hline$\geq 18$ & 41 & 15 & 26 & \\
\hline$<18$ & 22 & 10 & 12 & \\
\hline Tumor location & & & & 0.065 \\
\hline Tibia/femur & 39 & 12 & 27 & \\
\hline Other & 24 & 13 & 11 & \\
\hline Clinical stage & & & & $0.028^{\mathrm{a}}$ \\
\hline IIA & 45 & 14 & 31 & \\
\hline IIB/III & 18 & 11 & 7 & \\
\hline Tumor size (cm) & & & & 0.274 \\
\hline$\geq 8$ & 28 & 9 & 19 & \\
\hline$<8$ & 35 & 16 & 19 & \\
\hline Distant metastasis & & & & $0.011^{\mathrm{a}}$ \\
\hline Present & 19 & 3 & 16 & \\
\hline Absent & 44 & 22 & 22 & \\
\hline
\end{tabular}

${ }^{\mathrm{a}} \mathrm{P}<0.05 . \mathrm{miR}$, microRNA.

apoptosis, according to the manufacturer's protocol. Following harvesting and washing twice with PBS, the cells were stained with Annexin V-FITC and propidium iodide (PI), and incubated for $15 \mathrm{~min}$ at room temperature in the dark. Cell apoptosis was subsequently detected using a FACScan flow cytometer (Beckman Coulter, Inc., Brea, CA, USA).

Cell cycle distribution assay. After $48 \mathrm{~h}$ of transfection, U2OS and MG63 cells were harvested, washed twice with PBS and fixed with $70 \%$ ethanol at $4^{\circ} \mathrm{C}$ overnight, washed with PBS twice and incubated with $100 \mu \mathrm{l}$ RNaseA at $37^{\circ} \mathrm{C}$ in dark for 25 min. The cells were washed with PBS and stained with PI staining solution $(50 \mu \mathrm{g} / \mathrm{ml}$; containing $1 \mathrm{mg} / \mathrm{ml}$ RNase A and $0.1 \%$ Triton X-100 in PBS). Finally, the cell cycle distribution was assessed with a BD FACSCalibur flow cytometer (BD Biosciences, San Jose, CA, USA). Experiments were performed in triplicate.

Cell invasion Transwell assays. A cell invasion assay was conducted in a 24-well plate with Transwell chamber inserts (pore size, $8 \mathrm{~mm}$; Corning Incorporated, Corning, NY, USA). Transfected cells $\left(1 \times 10^{5}\right.$ cells) were placed into the upper chamber with Matrigel (BD Biosciences), respectively. The lower chamber was loaded with medium supplemented with $10 \%$ FBS. Following incubation for $48 \mathrm{~h}$, the cells on the upper surface of the membrane were removed, while the invading cells on the bottom surface of the Transwell chambers were fixed with methanol for $20 \mathrm{~min}$ at room temperature and stained with $0.1 \%$ crystal violet for $30 \mathrm{~min}$. Images of the stained cells were captured, and the number of cells was counted under an inverted microscope (Olympus Corporation, Tokyo, Japan). The results were averaged among three independent experiments.

Wound-healing assay. U2OS and MG63 cells (2x10 5 per well) were plated onto 6-well plates and cultured until confluence of $\sim 90 \%$ was reached. After $24 \mathrm{~h}$ of transfection, the plates were scrapped with a $10 \mu \mathrm{l}$ pipet tip, and the medium was replaced with fresh serum-free medium. Initial images were acquired as a reference, and further images of the previously photographed region were collected after $48 \mathrm{~h}$. The percentage of wound-healing rate $=[($ width at $0 \mathrm{~h}$-width at $48 \mathrm{~h}) /$ width at $0 \mathrm{~h}]$ $\mathrm{x} 100$.

Bioinformatics analysis and luciferase reporter assay. The miRNA target prediction websites, including PicTar (https://pictar.mdc-berlin.de), TargetScan (http://targetscan. org) and miRDB (http://www.mirdb.org) were used to search for the putative targets of miR-330-5p. Luciferase complexes were constructed by ligating oligonucleotides containing the wild-type (wt) or mutated (mut) putative target site of the survivin 3'-untranslated region (3'UTR) into the multi-cloning site of the pGL3 luciferase reporter vector (Promega Corporation, Madison, WI, USA). Subsequently, 293T cells ( $1 \times 10^{5}$ cells/well) were plated in a 24-well plate and co-transfected with $80 \mathrm{ng}$ of pGL3-survivin-3'-UTR (wt) or pGL3-survivin-3'-UTR (mut) and $50 \mathrm{nmol} / \mathrm{l}$ of the mimics or inhibitors (including miR-330-5p mimics, mimics NC, miR-330-5p inhibitor and inhibitor NC) using Lipofectamine ${ }^{\circledR} 2000$, according to the manufacturer's 
A

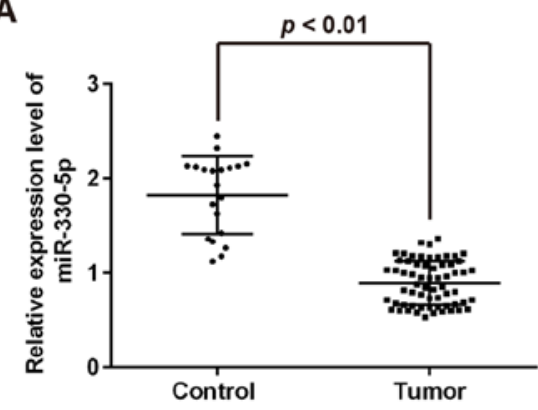

C

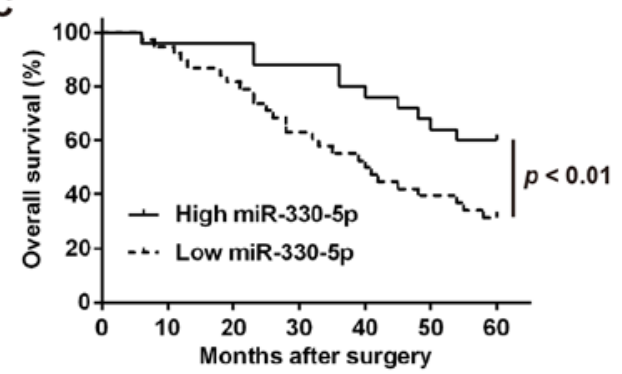

B

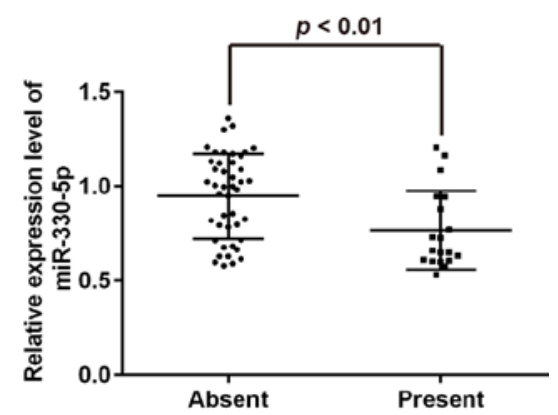

D

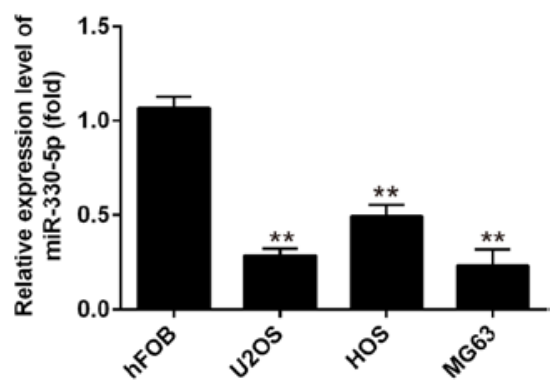

Figure 1. miR-330-5p is downregulated in OS tissues and cell lines. (A) miR-330-5p expression was measured by RT-qPCR in tumor tissues (n=63) and control osteochondroma tissues ( $\mathrm{n}=20)$. (B) miR-330-5p expression was measured in OS tissues with or without distant metastasis by RT-qPCR. (C) Kaplan-Meier survival curve demonstrated the association between the overall survival of osteosarcoma patients and the miR-330-5p levels. (D) miR-330-5p expression was detected in the HOS, U2OS and MG63 cells, and normal hFOB1.19 cells were used as the control. Data represent the mean \pm standard deviation of three independent experiments. ${ }^{* *} \mathrm{P}<0.01$ vs. hFOB1.19 cells. miR, microRNA; OS, osteosarcoma; RT-qPCR, reverse transcription-quantitative polymerase chain reaction.

protocol. Cells were transfected for $48 \mathrm{~h}$ at $37^{\circ} \mathrm{C}$, then luciferase activities were measured using the Dual-Luciferase Reporter Assay System (Promega Corporation). To correct for differences in transfection and harvesting efficiencies, Renilla luciferase activity was used to normalize the firefly luciferase activity.

Western blot analysis. Total protein was extracted from the cells using radioimmunoprecipitation assay lysis buffer (Beyotime Institute of Biotechnology, Shanghai, China), and the total cellular protein concentration was measured using a BCA assay kit (Pierce; Thermo Fisher Scientific, Inc.). Next, total protein samples $(40 \mu \mathrm{g})$ were separated by $8 \%$ SDS-PAGE and subsequently transferred to polyvinylidene difluoride membranes (GE Healthcare, Chicago, IL, USA) by electroblotting. Primary antibodies against survivin (cat no. ab76424; 1:5,000; Abcam, Cambridge, MA, USA) and $\beta$-actin (cat no. sc-58673; 1:2,000; Santa Cruz Biotechnology, Inc., Dallas, TX, USA) were then probed with the proteins on the membrane overnight at $4^{\circ} \mathrm{C}$. Following further incubation with secondary antibodies (cat no. 7076; 1:10,000; Cell Signaling Technology, Inc., Danvers, MA, USA) at $37^{\circ} \mathrm{C}$ for $1 \mathrm{~h}$. Protein bands were visualized with an Enhanced Chemiluminescence kit (GE Healthcare). Finally, ImageJ software (version 1.46; National Institutes of Health, Bethesda, MD, USA) was used to assess the intensity of the bands of interest; the endogenous control $\beta$-actin was used to normalize the expression of the selected genes.

Statistical analysis. Statistical analysis was performed using the SPSS program (version 18.0; SPSS, Inc., Chicago, IL, USA). Data are presented as the mean \pm standard deviation. Student's t-test or one-way analysis of variance followed by Tukey's post hoc test was used to analyze the difference among two or more than two sample groups, respectively. Spearman's analysis was used to calculate the correlation between the expression of miR-330-5p and survivin. The survival rate of patients was determined using the Kaplan-Meier method, and differences between groups were examined using the log-rank test. $\mathrm{P}<0.05$ was considered to denote a difference that was statistically significant.

\section{Results}

Expression of miR-330-5p and its diagnostic value in $O S$ tissues and cells. To investigate the potential role of miR-330-5p in OS, the expression of miR-330-5p was first determined in 63 OS tumor and 20 benign osteochondroma (control) tissues by RT-qPCR assay. As shown Fig. 1A, compared with the control group, miR-330-5p was significantly downregulated in OS tissues. Furthermore, the expression of miR-330-5p was markedly lower in tumors with distant metastasis as compared with that in tumors without distant metastasis (Fig. 1B), indicating that miR-330-5p downregulation is associated with OS metastasis. To further determine the clinical value of miR-330-5p, the patients were divided into the high miR-330-5p expression group ( $n=25)$ and low expression group $(n=38)$, using the median value of miR-330-5p expression in patients OS as a cutoff point (0.997). The results demonstrated that low miR-330-5p expression was associated with the clinical stage and distant metastasis, but not with age, gender, tumor location and tumor size in the OS patients (Table I). Compared with the patients 
A

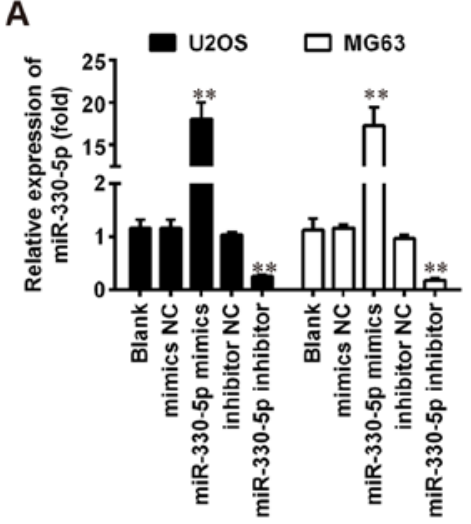

B

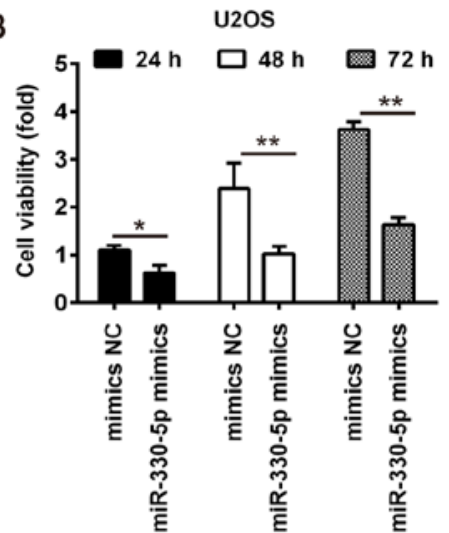

C

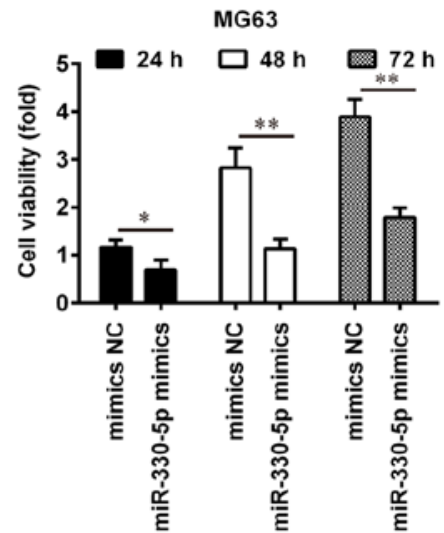

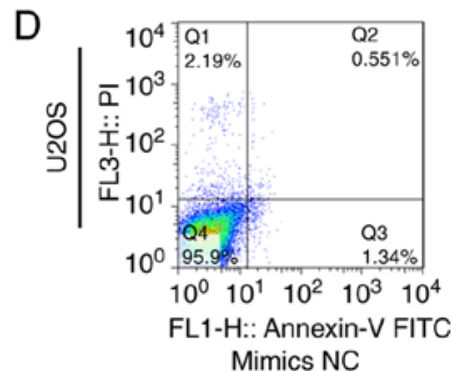
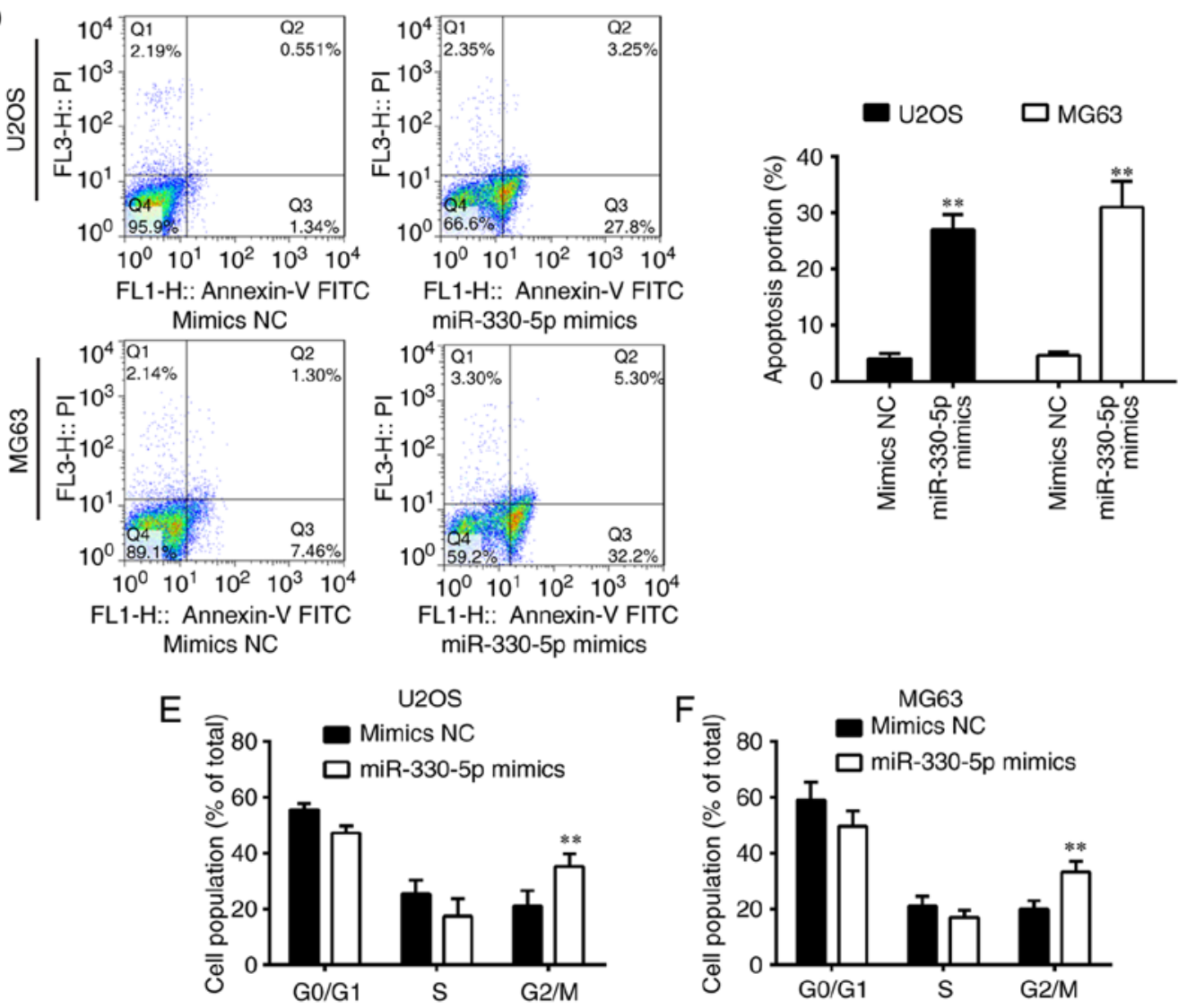

Figure 2. Overexpression of miR-330-5p suppressed cell viability, promoted cell apoptosis and induced cell cycle arrest. (A-F) U2OS and MG63 cells were transfected with $50 \mathrm{nM}$ miR-330-5p mimics, inhibitors, mimics NC or inhibitor NC, and used for subsequent analyses. (A) Transfection efficiency was assessed by reverse transcription-quantitative polymerase chain reaction after transfection for $48 \mathrm{~h}$; ${ }^{* *} \mathrm{P}<0.01 \mathrm{vs}$. Blank group. (B) U2OS and (C) MG63 cell viability was measured by Cell Counting Kit-8 assay at 24, 48 or $72 \mathrm{~h}$. (D) Cell apoptosis was detected by flow cytometry after transfection for $48 \mathrm{~h}$. Flow cytometry was also performed to observe the cell cycle distribution in (E) U2OS and (F) MG63 cells after transfection for $48 \mathrm{~h}$. Data represent the mean \pm standard deviation of three independent experiments. ${ }^{*} \mathrm{P}<0.05$ and ${ }^{* *} \mathrm{P}<0.01$ vs. mimics $\mathrm{NC}$ group. miR, microRNA; NC, negative control.

in the high miR-330-5p expression group, patients in the low miR-330-5p expression group had a significantly lower 5 -year overall survival rate (Fig. 1C). Taken together, these findings indicated that miR-330-5p may serve as an effective biomarker for the prognosis of patients with OS.

miR-330-5p expression levels were also measured in the HOS, U2OS and MG63 OS cell lines. In accordance with the results in OS tissues, the expression levels of miR-330-5p were significantly downregulated in the HOS, U2OS and MG63 cells, as compared with those in normal hFOB1.19 cells (Fig. 1D). U2OS and MG63 cells were selected for use in further experiments since they exhibited the lowest expression of miR-330-5p.

Overexpression of miR-330-5p inhibits OS cell viability, promotes cell apoptosis and induces cell cycle arrest. The downregulation of miR-330-5p in OS tissues suggested that miR-330-5p may function as a tumor suppressor in OS progression. To test this hypothesis, miR-330-5p mimics or inhibitor were transfected into U2OS and MG63 cells. Following miR-330-5p transfection, the expression level of miR-330-5p in the OS cell lines was significantly increased 
A

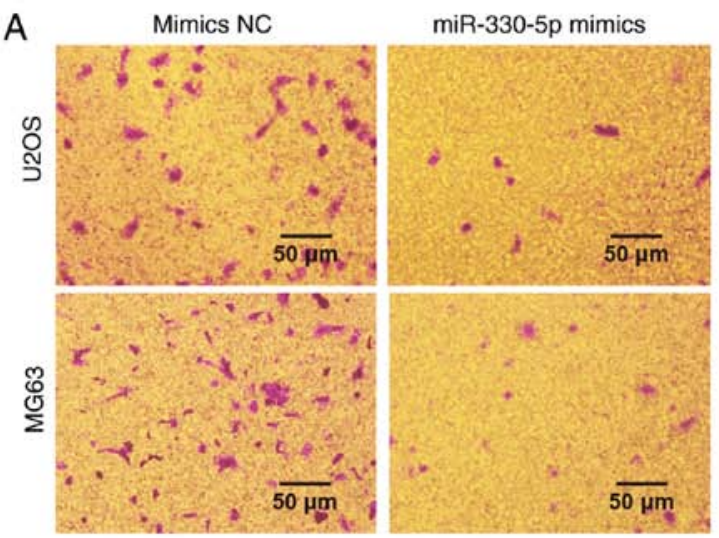

C

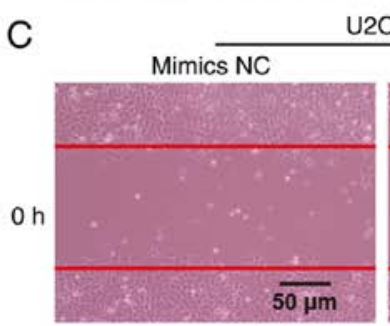

U2OS
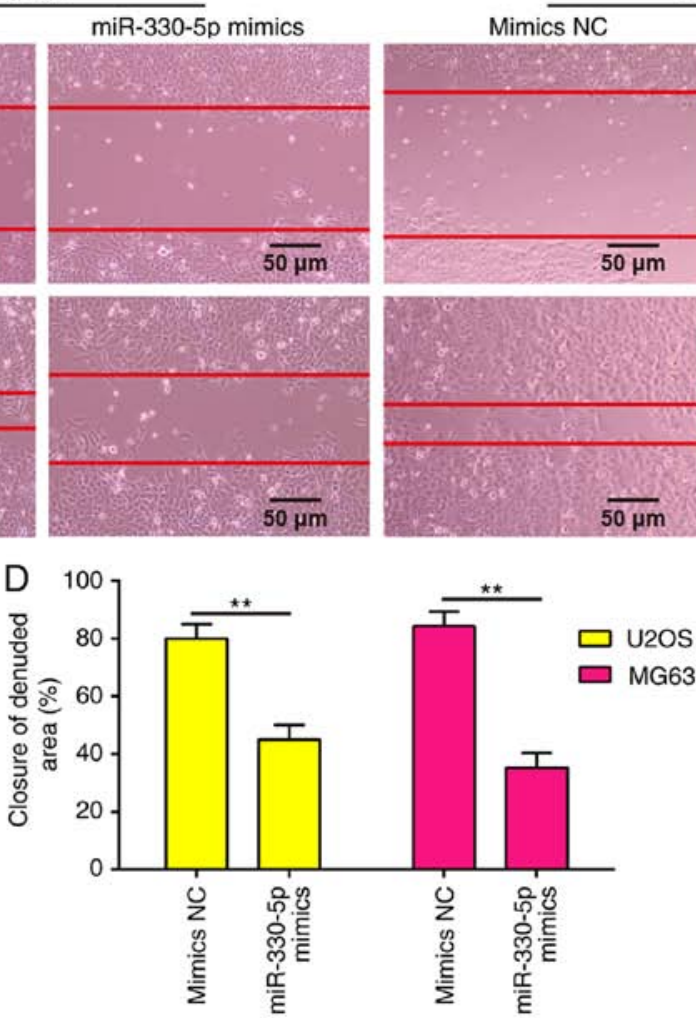

B
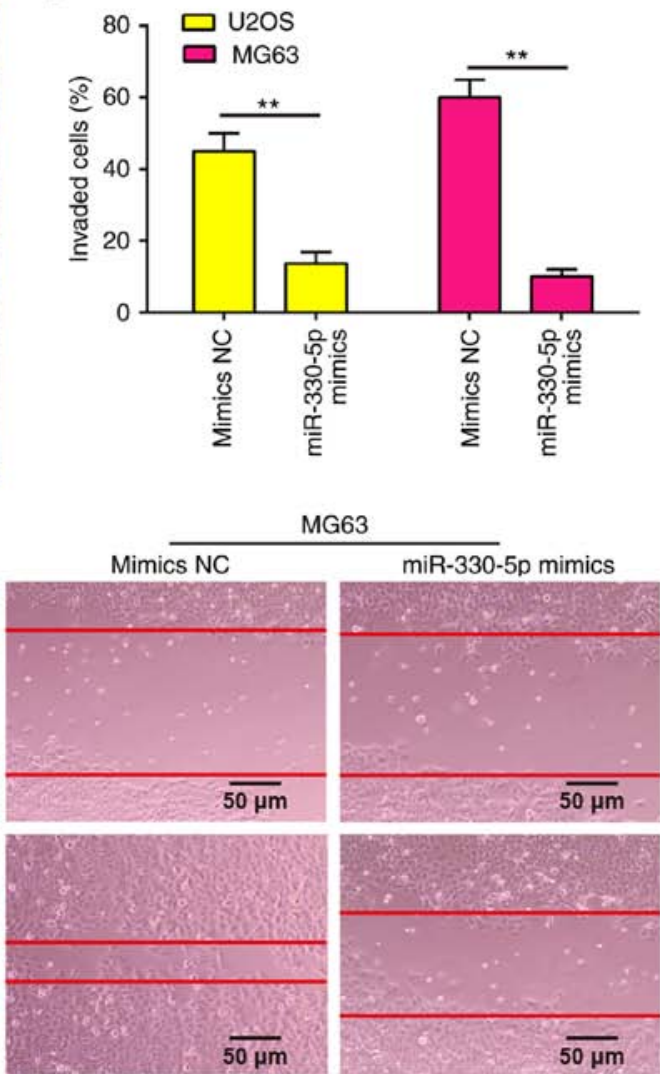

Figure 3. Overexpression of miR-330-5p suppressed cell invasion and migration. U2OS and MG63 cells were transfected with the 50 nM miR-330-5p mimics or mimics-NC for $48 \mathrm{~h}$, and then used for analysis. (A) Wound healing assay and (B) quantified migration abilities of U2OS and MG63 cells. (C) Transwell invasion assay and (D) quantified invasion abilities of U2OS and MG63 cells. Data represent the mean \pm standard deviation of three independent experiments. ${ }^{* *} \mathrm{P}<0.01$. miR, microRNA; NC, negative control.

when compared with that in the control groups (Fig. 2A). Conversely, miR-330-5p expression levels were significantly decreased in miR-330-5p inhibitor-transfected cells compared with inhibitor NC-transfected cells (Fig. 2A). Next, the effect of miR-330-5p overexpression on cell viability was examined by a CCK-8 assay. The results revealed that overexpression of miR-330-5p significantly suppressed the viability of U2OS and MG63 cells, as compared with that in the mimics NC group (Fig. 2B and C). Furthermore, the overexpression of miR-330-5p significantly promoted the apoptosis of U2OS and MG63 cells (Fig. 2D). Cell cycle analysis was further performed to demonstrate the underlying mechanisms by which miR-330-5p affected the cell viability. Flow cytometric analysis revealed that the overexpression of miR-330-5p in U2OS and MG63 cells significantly increased the number of cells at $G_{2} / M$ phase
(Fig. 2E and F). Taken together, these results suggested that miR-330-5p inhibited cell proliferation by inducing apoptosis and cell cycle arrest at $\mathrm{G}_{2} / \mathrm{M}$ phase.

Overexpression of miR-330-5p inhibits the invasion and migration abilities of $O S$ cells. Since the metastatic ability of OS is a critical factor in the poor prognosis of patients, the present study examined whether miR-330-5p is able to modulate the metastatic ability of OS cells using Transwell invasion and wound healing assays. As shown in Fig. 3A and B, overexpression of miR-330-5p in U2OS and MG63 cells significantly decreased the cell invasion ability as compared with the mimics NC group. Similarly, the wound healing assay demonstrated that overexpression of miR-330-5p clearly decreased the cell migration distance 
A

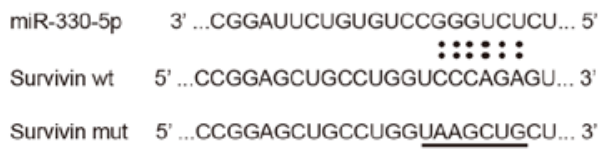

B

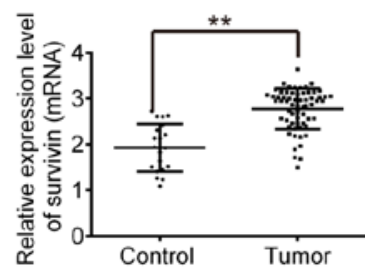

C

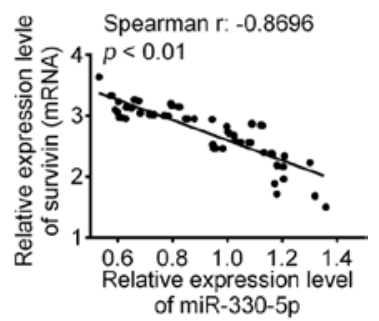

D

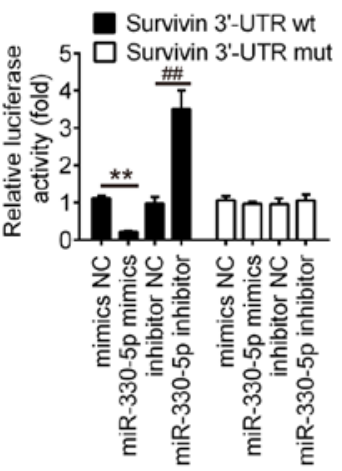

E

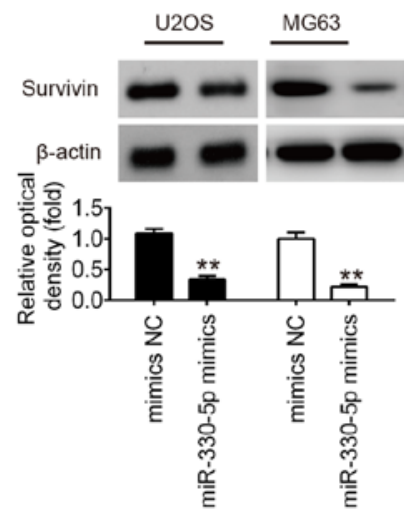

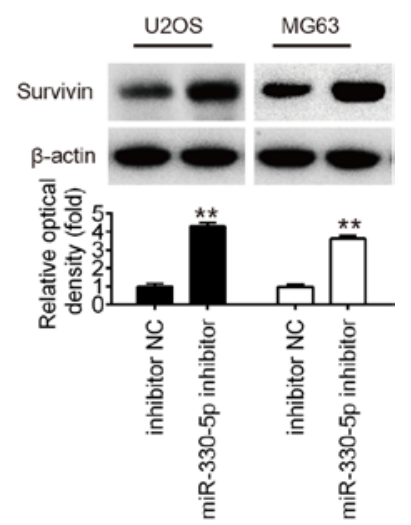

Figure 4. Survivin is a direct target of miR-330-5p. (A) Schematic of the survivin 3'-UTR containing the miR-330-5p binding site. (B) Survivin expression was measured by reverse transcription-quantitative polymerase chain reaction in OS tissues $(n=63)$ and osteochondroma tissues $(n=20)$. (C) Survivin expression was negatively associated with miR-330-5p expression in OS tissues $(r=-0.8696, P<0.01)$. (D) 293T cells were co-transfected with $80 \mathrm{ng}$ survivin-3'-UTR wt or mut reporter plasmids, and with $50 \mathrm{nM}$ miR-330-5p mimics, mimics NC, miR-330-5p inhibitor or inhibitor NC for $48 \mathrm{~h}$, and relative luciferase activity was measured. (E) U2OS and (F) MG63 cells were transfected with the $50 \mathrm{nM}$ miR-330-5p mimics, mimics-NC, miR-330-5p inhibitor or inhibitor NC for $48 \mathrm{~h}$, and then survivin protein expression was determined by western blot analysis. Data represent the mean \pm standard deviation of three independent experiments. ${ }^{* *} \mathrm{P}<0.01$. miR, microRNA; OS, osteosarcoma; NC, negative control; 3'-UTR, 3'-untranslated region; wt, wild-type; mut, mutant.

as compared with that of cells transfected with negative controls (Fig. 3C and D). Collectively, these data suggested that miR-330-5p suppressed the metastatic ability of OS cells.

Survivin is a direct target of miR-330-5p. Several studies have reported that miR-330-5p is involved in tumor progression via targeting the 3 '-UTR of its target genes $(11,14)$, including MUC1,ITGA5 and Pdia3 (17). To identify new potential target genes of miR-330-5p, the databases PicTar, TargetScan and miRBase were searched for candidate genes. Bioinformatics analysis revealed a miR-330-5p binding site in the 3'-UTR of survivin (Fig. 4A). In addition, it was observed that the mRNA level of survivin was significantly upregulated in OS tissues compared with the control group (Fig. 4B), and miR-330-5p expression was inversely correlated with survivin expression in OS tissues ( $r=-0.8696$; $\mathrm{P}<0.01$; Fig. 4C). To validate whether the 3'-UTR of survivin is a functional target of miR-330-5p, a luciferase reporter assay was performed. Compared with the NC group, introduction of miR-330-5p mimics markedly inhibited the relative luciferase activity of cells co-transfected with the survivin-3'-UTR wt, while miR-330-5p inhibitor significantly increased the luciferase activity of the wt group. However, the luciferase activity of the reporters containing the mut binding site exhibited no evident changes (Fig. 4D). To further investigate whether miR-330-5p regulates survivin expression, the effect of miR-330-5p overexpression or inhibition on survivin protein expression was examined in OS cells. It was clearly observed that overexpression of miR-330-5p inhibited the protein expression of survivin in the U2OS and MG63 cells, whereas knockdown of miR-330-5p promoted survivin expression (Fig. 4E and F). These results demonstrated that miR-330-5p was able to suppress the expression of the proto-oncogene survivin in OS cells by directly targeting the survivin 3'-UTR.

miR-330-5p suppresses cell growth and induces cell apoptosis through targeting survivin. First, the overexpression efficiency of pcDNA-survivin transfection in OS cells was determined (Fig. 5A); the expression levels of survivin in was significantly higher compared with empty vector-transfected cells, which indicated successful transfection of the overexpression vector. To investigate whether miR-330-5p exerts an antitumor effect through regulating survivin, U2OS and MG63 cells were co-transfected with miR-330-5p mimics and pcDNA-survivin plasmid, and cell viability, apoptosis, invasion and migration was assessed. As shown in Fig. 5B, the transfection of cells with survivin expression vectors significantly upregulated survivin expression and restored the decreased survivin expression in miR-330-5p mimics-transfected cells. CCK-8 assay and flow cytometry assays were then conducted to determine the cell viability and apoptosis, respectively. The results demonstrated that overexpression of survivin significantly increased the viability and inhibited apoptosis in miR-330-5p mimics + pcDNA-vector-transfected cells (Fig. 5C and D). Furthermore, the inhibitory effects of miR-330-5p overexpression on the invasion and migration abilities of cells were reversed by survivin overexpression (Fig. 5E and F). These results suggest that miR-330-5p functions as a tumor suppressor in OS, at least partially, through regulating survivin expression. 
A

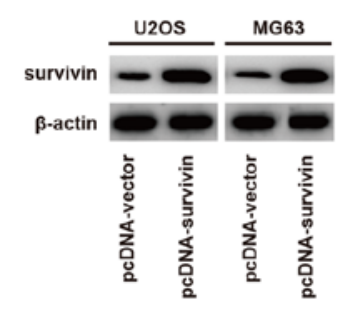

D

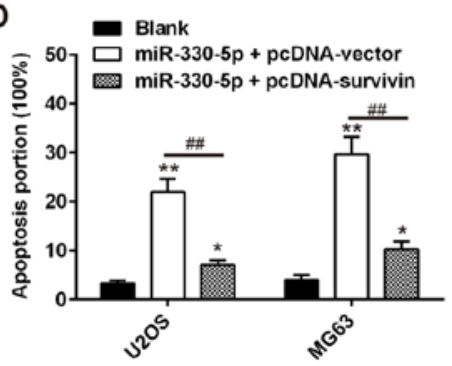

B

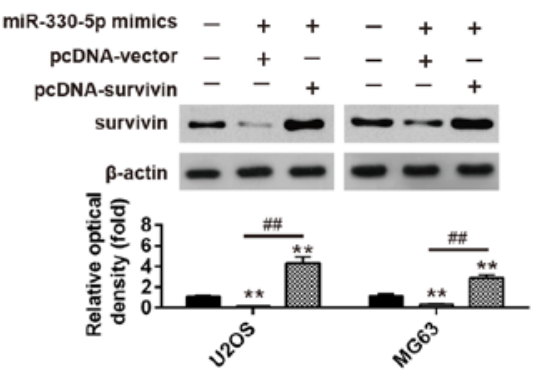

E

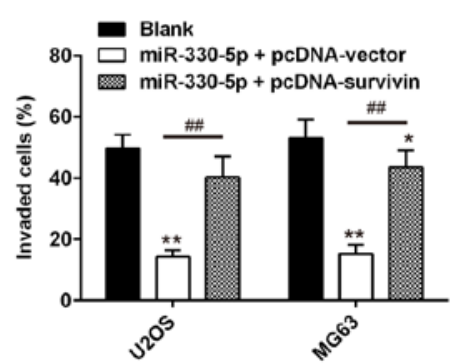

C

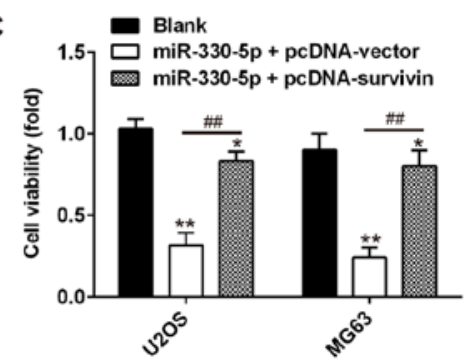

$\mathbf{F}$

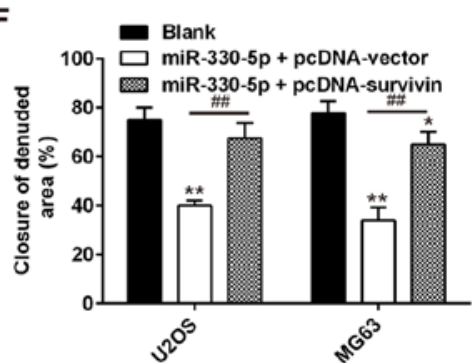

Figure 5. miR-330-5p inhibited cell viability and induced cell apoptosis by targeting survivin. (A) U2OS and MG63 cells were transfected with $2 \mu \mathrm{g}$ pcDNA-survivin or pcDNA-vector for $48 \mathrm{~h}$, and the overexpression efficiency was detected by western blot analysis. (B) U2OS and MG63 cells were co-transfected with the $2 \mu \mathrm{g}$ pcDNA-survivin and $50 \mathrm{nM}$ miR-330-5p mimics for $48 \mathrm{~h}$, and then used for analysis. Western blot analysis was used to detect the survivin protein level after $48 \mathrm{~h}$ of co-transfection. (C) Viability of U2OS and MG63 cells was measured by Cell Counting Kit- 8 assay after 48 h of co-transfection. (D) Apoptosis was detected by flow cytometry after $48 \mathrm{~h}$ of co-transfection. (E) Migration ability of U2OS and MG63 cells, as measured by the wound healing assay after $48 \mathrm{~h}$ of co-transfection. (F) Invasion ability of U2OS and MG63 cells, as measured by the Transwell invasion assay after $48 \mathrm{~h}$ of co-transfection. Data represent the mean \pm standard deviation of three independent experiments. ${ }^{*} \mathrm{P}<0.05$ and ${ }^{* *} \mathrm{P}<0.01$ vs. Blank group in U2OS or MG63 cells; ${ }^{\# \# ~} \mathrm{P}<0.01$ vs. miR-330-5p + pcDNA-vector group in U2OS or MG63 cells. miR, microRNA; NC, negative control.

\section{Discussion}

In the present study, the results indicated that miR-330-5p was significantly downregulated in OS tissues and cell lines, and its low expression was closely associated with the clinical stage and overall survival of OS patients. Subsequently, functional experiments in OS cell lines revealed that overexpression of miR-330-5p inhibited the cell viability, migration and invasion, promoted cell apoptosis and induced cell cycle arrest. Mechanism research demonstrated that the underlying mechanism of miR-330-5p was associated with targeting and inhibiting survivin, a well-known oncogenic gene. Overall, the current study provided an insight into the possible involvement of the miR-330-5p/survivin axis in the development and progression of OS.

Recent publications have demonstrated that miR-330-5p functions as a tumor suppressor or an oncogene in the context of different cancer types. For instance, Kong et al (18) reported that miR-330-5p was significantly decreased in NSCLC, while overexpression of miR-330-5p markedly inhibited NSCLC cell growth and promoted cell apoptosis. Lee et al (19) also demonstrated that miR-330 functioned as a tumor suppressor and induced the apoptosis of prostate cancer cells through targeting E2F1. By contrast, miR-330 was found to be upregulated in glioblastoma and to function as an oncogenic factor by enhancing proliferation and invasion, and inhibiting apoptosis through the activation of ERK and phosphoinositide 3-kinase/protein kinase B pathways $(20,21)$. However, the expression and role of miR-330-5p in OS remain unknown. In the current study, miR-330-5p was significantly downregulated in OS tissues, with a markedly lower expression detected in metastatic tissues, indicating that decreased miR-330-5p was associated with OS metastasis. The study also identified that low miR-330-5p expression in OS patients was significantly associated with the clinical stage and distant metastasis, as well as poorer prognosis. These findings suggested that miR-330-5p may serve as an effective biomarker for the prognosis of patients with OS. Next, the role of miR-330-5p in malignant progression of OS was examined. In OS cells, overexpression of miR-330-5p significantly inhibited the cell proliferation, promoted cell apoptosis and induced cell cycle arrest at $\mathrm{G} 2 / \mathrm{M}$ phase in vitro. It was also observed that the migration and invasion of OS cells were suppressed by miR-330-5p overexpression, which suggests that miR-330-5p overexpression exerts an antitumor effect in OS.

Survivin, the most important member of the inhibitor of apoptosis family, is known to serve important roles in tumor cell proliferation and invasion, therapeutic resistance and poor prognosis $(22,23)$. For instance, a previous study reported that survivin was apparently overexpressed in ovarian cancer, and its overexpression induced cell proliferation and angiogenesis (24). Wang and Ye (25) reported that interfering with the expression of survivin was able to inhibit the cell proliferation, migration and invasion, and promote apoptosis in breast cancer cells. These previous findings suggested that survivin exerts an oncogenic role in tumorigenesis. Recently, Chen et al (26) demonstrated that miR-34a and miR-203 repressed the proliferation of human OS cells by downregulating the expression of survivin. In the present study, the results identified survivin as a direct and functional target of miR-330-5p, and miR-330-5p was found to directly negatively regulate survivin expression in OS cells. Furthermore, it was demonstrated that survivin was highly expressed in OS tissues, and a significant negative correlation was observed between miR-330-5p and survivin expression. Notably, overexpression of survivin partially reversed the 
inhibitory effects of miR-330-5p in OS cells. These data suggest that miR-330-5p exerts its tumor suppressive role by targeting survivin.

In conclusion, the data of the present study revealed that miR-330-5p functions as a tumor suppressor, and participates in the inhibition of cell proliferation and invasion, as well as promotes apoptosis and induces cell cycle arrest in OS cells by directly regulating the expression of survivin. These findings indicated that miR-330-5p may be a promising therapeutic target for the treatment of OS.

\section{Acknowledgements}

Not applicable.

\section{Funding}

No funding was received.

\section{Availability of data and materials}

All data generated or analyzed during the present study are included in this published article.

\section{Authors' contributions}

HW and LL performed the experiments, contributed to data analysis and wrote the paper. SF designed the study and contributed to data analysis and experimental materials. All authors read and approved the final manuscript.

\section{Ethics approval and consent to participate}

All participants provided informed consent for the use of human specimens for clinical research. The present study was approved by the Anhui Provincial Hospital, Anhui Medical University (Hefei, China).

\section{Patient consent for publication}

Not applicable.

\section{Competing interests}

The authors declare that they have no competing interests.

\section{References}

1. Kobayashi E, Hornicek FJ and Duan Z: MicroRNA involvement in osteosarcoma. Sarcoma 2012: 359739, 2012.

2. Bruland OS, Bauer H, Alvegaard T and Smeland S: Treatment of osteosarcoma. The Scandinavian Sarcoma Group experience. Cancer Treat Res 152: 309-318, 2009.

3. Ambros V: The functions of animal microRNAs. Nature 431: 350-355, 2004

4. Lagos-Quintana M, Rauhut R, Lendeckel W and Tuschl T: Identification of novel genes coding for small expressed RNAs. Science 294: 853-858, 2001.

5. Huang Y, Shen XJ, Zou Q, Wang SP, Tang SM and Zhang GZ: Biological functions of microRNAs: A review. J Physiol Biochem 67: 129-139, 2011.

6. Sotillo E and Thomas-Tikhonenko A: Shielding the messenger (RNA): microRNA-based anticancer therapies. Pharmacol Ther 131: 18-32, 2011
7. Peng N, Miao Z, Wang L, Liu B, Wang G and Guo X: MiR-378 promotes the cell proliferation of osteosarcoma through down-regulating the expression of Kruppel-like factor 9. Biochem Cell Biol 96: 515-521, 2018.

8. Liu Z, Li Q, Zhao X, Cui B, Zhang L and Wang Q: MicroRNA-935 inhibits proliferation and invasion of osteosarcoma cells by directly targeting High mobility group box 1. Oncol Res: Feb 22, 2018.

9. Yang X, Wang L, Wang Q, Li L, Fu Y and Sun J: MiR-183 inhibits osteosarcoma cell growth and invasion by regulating LRP6-Wnt/ $\beta$-catenin signaling pathway. Biochem Biophys Res Commun 496: $1197-1203,2018$.

10. Dong J, Liu Y, Liao W, Liu R, Shi P and Wang L: miRNA-223 is a potential diagnostic and prognostic marker for osteosarcoma. J Bone Oncol 5: 74-79, 2016.

11. Feng L, Ma J, Ji H, Liu Y and Hu W: miR-330-5p suppresses glioblastoma cell proliferation and invasiveness through targeting ITGA5. Biosci Rep 37: pii: BSR20170019, 2017.

12. Trehoux S, Lahdaoui F, Delpu Y, Renaud F, Leteurtre E, Torrisani J, Jonckheere $\mathrm{N}$ and Van Seuningen I: Micro-RNAs miR-29a and miR-330-5p function as tumor suppressors by targeting the MUC1 mucin in pancreatic cancer cells. Biochim Biophys Acta 1853: 2392-2403, 2015

13. Wang H, Chen SH, Kong P, Zhang LY, Zhang LL, Zhang NQ and $\mathrm{Gu} \mathrm{H}$ : Increased expression of miR-330-3p: A novel independent indicator of poor prognosis in human breast cancer. Eur Rev Med Pharmacol Sci 22: 1726-1730, 2018

14. Su BB, Zhou SW, Gan CB and Zhang XN: MiR-330-5p regulates tyrosinase and PDIA3 expression and suppresses cell proliferation and invasion in cutaneous malignant melanoma. J Surg Res 203: 434-440, 2016

15. Wei CH, Wu G, Cai Q, Gao XC, Tong F, Zhou R, Zhang RG, Dong JH, Hu Y and Dong XR: MicroRNA-330-3p promotes cell invasion and metastasis in non-small cell lung cancer through GRIA3 by activating MAPK/ERK signaling pathway. J Hematol Oncol 10: 125, 2017

16. Livak KJ and Schmittgen TD: Analysis of relative gene expression data using real-time quantitative PCR and the 2(-Delta Delta C(T)) method. Methods 25: 402-408, 2001.

17. Kim BK, Yoo HI, Choi K and Yoon SK: miR-330-5p inhibits proliferation and migration of keratinocytes by targeting Pdia3 expression. FEBS J 282: 4692-4702, 2015.

18. Kong R, Liu W, Guo Y, Feng J, Cheng C, Zhang X, Ma Y, Li S, Jiang $\mathrm{J}$, Zhang J, et al: Inhibition of NOB1 by microRNA-330-5p overexpression represses cell growth of non-small cell lung cancer. Oncol Rep 38: 2572-2580, 2017.

19. Lee KH, Chen YL, Yeh SD, Hsiao M, Lin JT, Goan YG and Lu PJ: MicroRNA-330 acts as tumor suppressor and induces apoptosis of prostate cancer cells through E2F1-mediated suppression of Akt phosphorylation. Oncogene 28: 3360-3370, 2009.

20. Qu S, Yao Y, Shang C, Xue Y, Ma J, Li Z and Liu Y: MicroRNA-330 is an oncogenic factor in glioblastoma cells by regulating SH3GL2 gene. PLoS One 7: e46010, 2012.

21. Yao Y, Xue Y, Ma J, Shang C, Wang P, Liu L, Liu W, Li Z, Qu S, Li Z and Liu Y: MiR-330-mediated regulation of SH3GL2 expression enhances malignant behaviors of glioblastoma stem cells by activating ERK and PI3K/AKT signaling pathways. PLoS One 9: e95060, 2014.

22. Luk SU, Xue H, Cheng H, Lin D, Gout PW, Fazli L, Collins CC, Gleave ME and Wang Y: The BIRC6 gene as a novel target for therapy of prostate cancer: Dual targeting of inhibitors of apoptosis. Oncotarget 5: 6896-6908, 2014.

23. Kusner LL, Ciesielski MJ, Marx A, Kaminski HJ and Fenstermaker RA: Survivin as a potential mediator to support autoreactive cell survival in myasthenia gravis: A human and animal model study. PLoS One 9: e102231, 2014.

24. Wang HX, Chen G, Li GL and Jiang YJ: Expression and significance of Survivin and Smac in ovarian mucinous tumors. Zhonghua Bing Li Xue Za Zhi 39: 387-390, 2010 (In Chinese).

25. Wang $\mathrm{H}$ and Ye YF: Effect of survivin siRNA on biological behaviour of breast cancer MCF7 cells. Asian Pac J Trop Med 8: 225-228, 2015

26. Chen X, Chen XG, Hu X, Song T, Ou X, Zhang C, Zhang W and Zhang C: MiR-34a and miR-203 Inhibit survivin expression to control cell proliferation and survival in human osteosarcoma cells. J Cancer 7: 1057-1065, 2016.

This work is licensed under a Creative Commons Attribution-NonCommercial-NoDerivatives 4.0 International (CC BY-NC-ND 4.0) License. 